\title{
Nanopore-targeted sequencing for simultaneous diagnosis of suspected sepsis and early targeted therapy
}

\author{
Jun-Ying Li ${ }^{1}$, Gai-Gai Shen ${ }^{2}$, Tian-Gang Liu ${ }^{2}$, Liang V. Tang ${ }^{1} \wedge$, Ling-Hui Xia ${ }^{1}$, Yu Hu$^{1}$ \\ ${ }^{1}$ Institute of Hematology, Union Hospital, Tongji Medical College, Huazhong University of Science and Technology, Wuhan, China; ${ }^{2}$ Key \\ Laboratory of Combinatorial Biosynthesis and Drug Discovery, Ministry of Education and Wuhan University School of Pharmaceutical Sciences, \\ Wuhan, China \\ Correspondence to: Ling-Hui Xia, Liang V. Tang. Union Hospital, Tongji Medical College, Huazhong University of Science and Technology, No. 1277 \\ Jiefang Avenue, Wuhan 430022, China. Email: linghuixia@hust.edu.cn; lancet_tang@hust.edu.cn.
}

Submitted Jun 06, 2021. Accepted for publication Nov 02, 2021.

doi: 10.21037/atm-21-2923

View this article at: https://dx.doi.org/10.21037/atm-21-2923

Bloodstream infection can rapidly progress to sepsis with each delay to appropriate therapy. In particular, patients with advanced age, active cancer, hematological disease, immunocompromised status, or critical illness are prone to severe infection (1). The main characteristics of infection in these patients are extremely rapid progression and resistance to conventional broad-spectrum antibiotics. Early identification of pathogens is imperative for reducing the risk of death (2). However, standard diagnostic methods rely on culturing, which has poor sensitivity and is not suitable for atypical infections with fastidious organisms. Even with typical pathogenic microorganisms, the turnaround time ranges from 48 to 96 hours. Therefore, culturing is always too slow to guide targeted antimicrobial therapy.

Based on third-generation sequencing technology, we directly incorporated a panel of microbial tags and developed nanopore-targeted sequencing (NTS). Using real-time NTS, we successfully identified pathogens under complex host-microorganism settings, such as whole blood samples. This approach can reduce testing time and clinical turnaround time to less than 2 and 6 hours, respectively, enabling early targeted therapy.

The detailed protocol and evaluation of NTS have been published elsewhere as a methodological study $(3,4)$. Briefly, instead of a metagenomic analysis, a panel of 27,668 microbial tags (including bacteria, fungi, viruses, drugresistant genes, and atypical pathogens), were incorporated into the nanopore sequencing platform. Therefore, targeted sequencing, with full-length read and real-time analytic approaches, was developed. Pathogens with moderateto-high abundance were reported within 2 hours after sequencing, and pathogens with low abundance were reported within 8 hours after sequencing. Thus, a total clinical turnaround time of 6 to 18 hours can be achieved. Background noise was filtered out using negative controls. The cut-off value for a positive diagnosis was 20 reads. The limit of detection was 25 colony-forming units $/ \mathrm{mL}$.

This retrospective case series study was registered at the Chinese Clinical Trial Registry (www.chictr.org. cn, No. ChiCTR2000028904), and was approved by the Ethics Committee of the Union Hospital affiliated with the Huazhong University of Science and Technology (IRB approval ID: 2019-S316). Written informed consent was obtained from all patients to publish this study.

Eleven patients with hematologic disorders who underwent NTS diagnosis between November and December 2019 were consecutively enrolled and retrospectively reviewed. Ten patients underwent hematopoietic stem cell transplantation (Table S1). In 6 patients, the procalcitonin (PCT) or C-reactive protein (CRP) levels were more than $20 \mathrm{ng} / \mathrm{mL}$ or $100 \mathrm{mg} / \mathrm{L}$, respectively, during the first episode of fever. For each infection, routine microbiological culturing (Appendix 1) was performed at least once, while NTS was performed only once. NTS sampling was performed at the same time as the

^ ORCID: 0000-0002-3253-0220. 
culture sampling.

Traditional cultures were able to detect a pathogen in only 2 of the 11 cases. NTS identified pathogenic microorganisms or conditional pathogenic microorganisms in all 11 cases $(100 \%)$, with a clinical turnaround time within 6 hours in 9 cases. According to The Sanford Guide to Antimicrobial Therapy 2018 (5), appropriate antibiotics were immediately prescribed (Appendix 1). One patient died of severe sepsis (PCT >100 ng/mL) 23 hours after the first episode of hyperpyrexia; and the CRP level in the other 10 cases decreased steadily, and the infection was completely controlled (Table 1).

As an example, in case 1, a 15-year-old girl diagnosed with acute lymphoblastic leukemia, underwent haploidentical hematopoietic stem cell transplantation (Figure S1). Laminar-flow ward admission was used as the reference point for day 1. At first, she received preventive antimicrobial therapy (gentamicin + fluconazole + ganciclovir). After myeloablative conditioning, allogeneic transplantation of both the peripheral blood and bone marrow stem cells from a haploidentical donor (her father) was performed. Upon neutropenia, PCT and CRP levels rapidly increased to $51.8 \mathrm{ng} / \mathrm{mL}$ and $198 \mathrm{mg} / \mathrm{L}$, respectively. Because of sepsis, broad-spectrum antibiotics were empirically administered (cefoperazone + tegacycline + caspofungin). Four days later, the first NTS was performed, which revealed a bloodstream infection with Streptococcus mitis. The inflammatory markers decreased gradually after intravenous daptomycin treatment, and she recovered after granulocyte engraftment. However, recurrent hyperpyrexia and grade 4 oral mucositis occurred 2 days later. The second NTS revealed highly abundant, multidrug-resistant Pandoraea sputorum in both blood and pharyngeal swab samples within 6 hours after sampling (Figure S2). The antimicrobial strategy was immediately replaced with imipenem combined with sulfamethoxazole. Infection was eventually controlled on day 51 . No microorganisms were detected in additional NTS tests (data not shown). During the entire course of infection, no pathogens were detected in any of the 9 routine blood cultures.

Third-generation sequencing technology, including the Oxford Nanopore Technologies platform, is widely employed in metagenomics studies involving animal, plant, and microorganism samples (6-9). Nanopore sequencing may overcome many shortcomings faced by next-generation sequencing as a diagnostic tool. It can generate read-by-read data with individual read lengths of tens and thousands of nucleotides, by utilizing cost-effective and real-time longread sequencing strategy. Nanopore sequencing provides higher resolution, greater accuracy, and faster turnaround time, than do currently available commercial techniques. Therefore, its implementation in the clinical setting will markedly improve real-time point-of-care pathogen diagnosis (10-13). In one study, nanopore metagenomics was developed to enable the rapid diagnosis of bacterial lower respiratory infections using respiratory samples. Compared with culturing, the optimized method was $96.6 \%$ sensitive and $41.7 \%$ specific for bacteria detection, with a minimum turnaround time of 6 hours under experimental conditions (14). In contrast, we developed a novel NTS rather than the metagenomics method, and presented the first clinical report that real-time NTS can timely identify pathogens in whole blood samples from patients with severe infection. Targeted microbial tags enable pathogen detection in whole blood samples, a more complex hostmicroorganism setting, and further shorten the testing duration. In this study, NTS detected multiple pathogens including uncommon and atypical pathogens in each patient within 6 to 18 hours in a clinical context. As a result, these patients with severe infections benefited from early targeted antimicrobial therapy. Moreover, our method can be expanded to patients in different clinical settings.

In conclusion, NTS is a promising approach for rapidly and accurately characterizing pathogens, and guiding antimicrobial treatment. This application should be confirmed and optimized for future clinical practice. 
Table 1 Results of nanopore targeted sequencing testing and clinical effect

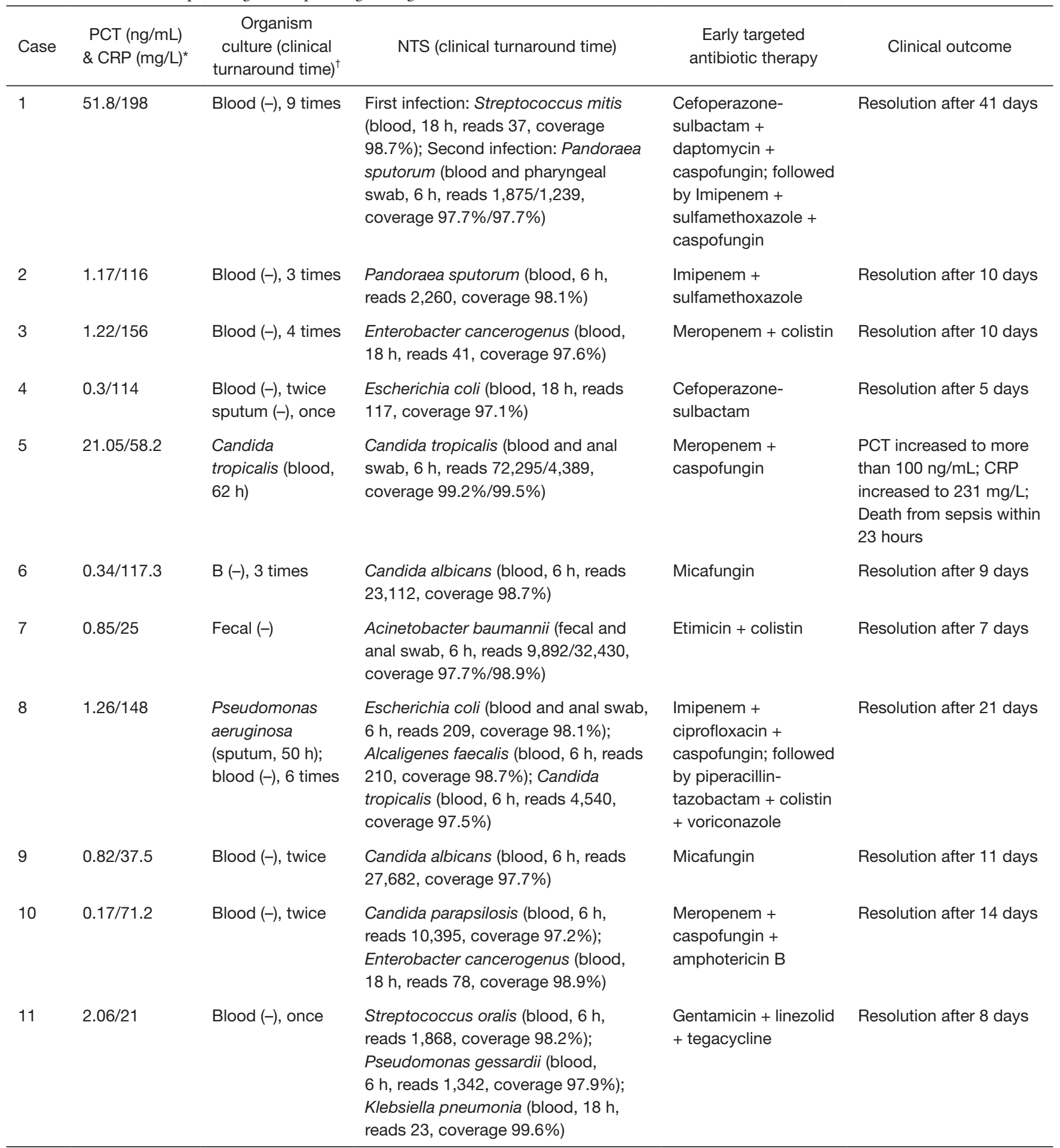

The prophylactic antibiotics in this setting of HSCT for each patient are "Moxifloxacin or Gentamicin" + oral "Fluconazole or Voriconazole". *, PCT and CRP tests at the first episode of fever; Normal levels: PCT $<0.5 \mathrm{ng} / \mathrm{mL}, \mathrm{CRP}<8 \mathrm{mg} / \mathrm{L} ;{ }^{\dagger}$, clinical turnaround time: from sampling to report. CRP, C-reactive protein; PCT, procalcitonin; NTS, nanopore targeted sequencing. 


\section{Acknowledgments}

We thank the 11 patients enrolled in the current study. Funding: This work was supported by the Program for HUST Academic Frontier Youth Team (No. 2018QYTD14) and the National Natural Science Foundation of China (No. 81973995). The listed funding organization had no role in this work.

\section{Footnote}

Provenance and Peer Review: This article was a standard submission to the journal. The article has undergone external peer review.

Conflicts of Interest: All authors have completed the ICMJE uniform disclosure form (available at https://dx.doi. org/10.21037/atm-21-2923). The authors have no conflicts of interest to declare.

Ethical Statement: The authors are accountable for all aspects of the work in ensuring that questions related to the accuracy or integrity of any part of the work are appropriately investigated and resolved. This retrospective case series study was registered at the Chinese Clinical Trial Registry (www.chictr.org.cn, No. ChiCTR2000028904), and was approved by the Ethics Committee of the Union Hospital affiliated with the Huazhong University of Science and Technology (IRB approval ID: 2019-S316). Written informed consent was obtained from all patients to publish this study.

Open Access Statement: This is an Open Access article distributed in accordance with the Creative Commons Attribution-NonCommercial-NoDerivs 4.0 International License (CC BY-NC-ND 4.0), which permits the noncommercial replication and distribution of the article with the strict proviso that no changes or edits are made and the original work is properly cited (including links to both the formal publication through the relevant DOI and the license). See: https://creativecommons.org/licenses/by-nc-nd/4.0/.

\section{References}

1. Li D, Zhang J, Han W, et al. Evaluation of the updated "Candida score" with Sepsis 3.0 criteria in critically ill patients. Ann Transl Med 2020;8:917.

2. Wang $\mathrm{L}, \mathrm{Ma} \mathrm{X}, \mathrm{He} \mathrm{H}$, et al. Compliance with the Surviving Sepsis Campaign guideline 1-hour bundle for septic shock in China in 2018. Ann Transl Med 2021;9:278.

3. Wang M, Fu AS, Hu B, et al. Same-Day Simultaneous Diagnosis of Bacterial and Fungal Infections in Clinical Practice by Nanopore Targeted Sequencing. MedRxiv 2020. doi:10.1101/2020.04.08.20057604

4. Huang Q, Fu A, Wang Y, et al. Microbiological diagnosis of endophthalmitis using nanopore targeted sequencing. Clin Exp Ophthalmol 2021;49:1060-8.

5. David NG, Henry FC, George ME, et al. The Sanford guide to antimicrobial therapy 2018 (48th edition). New York: Antimicrobial Therapy Inc., 2019.

6. Beaulaurier J, Luo E, Eppley JM, et al. Assembly-free single-molecule sequencing recovers complete virus genomes from natural microbial communities. Genome Res 2020;30:437-46.

7. Cao Y, Li J, Chu X, et al. Nanopore sequencing: a rapid solution for infectious disease epidemics. Sci China Life Sci 2019;62:1101-3.

8. Kafetzopoulou LE, Pullan ST, Lemey P, et al. Metagenomic sequencing at the epicenter of the Nigeria 2018 Lassa fever outbreak. Science 2019;363:74-7.

9. Chiu CY, Miller SA. Clinical metagenomics. Nat Rev Genet 2019;20:341-55.

10. Wang M, Fu A, Hu B, et al. Nanopore Targeted Sequencing for the Accurate and Comprehensive Detection of SARS-CoV-2 and Other Respiratory Viruses. Small 2020;16:e2002169.

11. Sheka D, Alabi N, Gordon PMK. Oxford nanopore sequencing in clinical microbiology and infection diagnostics. Brief Bioinform 2021;22:bbaa403.

12. Abril MK, Barnett AS, Wegermann K, et al. Diagnosis of Capnocytophaga canimorsus Sepsis by Whole-Genome Next-Generation Sequencing. Open Forum Infect Dis 2016;3:ofw144.

13. Sakamoto Y, Zaha S, Nagasawa S, et al. Long-read wholegenome methylation patterning using enzymatic base conversion and nanopore sequencing. Nucleic Acids Res 2021;49:e81.

14. Charalampous T, Kay GL, Richardson H, et al. Nanopore metagenomics enables rapid clinical diagnosis of bacterial lower respiratory infection. Nat Biotechnol 2019;37:783.

Cite this article as: Li JY, Shen GG, Liu TG, Tang LV, Xia LH, Hu Y. Nanopore-targeted sequencing for simultaneous diagnosis of suspected sepsis and early targeted therapy. Ann Transl Med 2021;9(23):1749. doi: 10.21037/atm-21-2923 


\section{Supplementary Methods}

\section{NTS}

A $2 \mathrm{~mL}$ blood sample was centrifuged at $200 \times \mathrm{g}$ for 2 minutes to remove blood cells, and the was analyzed using Oxford Nanopore MinION or GridION platform. The detailed protocol of NTS has been published as a methodological study in reference 3 of this letter.

\section{Blood Culture}

Blood samples were collected during a period of high fever (body temperature over $38.5{ }^{\circ} \mathrm{C}$ ) with chills. For each blood culture, a total of $40 \mathrm{~mL}$ of blood was collected from both a peripheral vein $(20 \mathrm{~mL})$ and a venous catheter $(20 \mathrm{~mL})$, and subjected to cultivation of aerobic and anaerobic bacteria, and fungi. Blood culturing was performed on an Automated Blood Culture System (Thermo Fisher Scientific, Waltham, MA, USA), according to the manufacturer's instructions.

\section{Selection of Antibiotics}

The institution-specific clinical guidelines for hematopoietic stem cell transplantation patients with febrile neutropenia are as follows: (1) neutropenia without fever, the prophylactic antibiotics are moxifloxacin or gentamicin + oral fluconazole or voriconazole; (2) after pathogen identification, the therapeutic antibiotics are prescribed according to The Sanford Guide to Antimicrobial Therapy; and (3) if no pathogen is identified, empirical antibiotics are prescribed according to the personal experience of the attending physician.

Since no antibiotic resistance mechanisms were detected by the NTS, we chose the antibiotics with the lowest likelihood of drug resistance according to The Sanford guide to antimicrobial therapy as well as drug-resistance data from the past 2 years in our local laboratory.

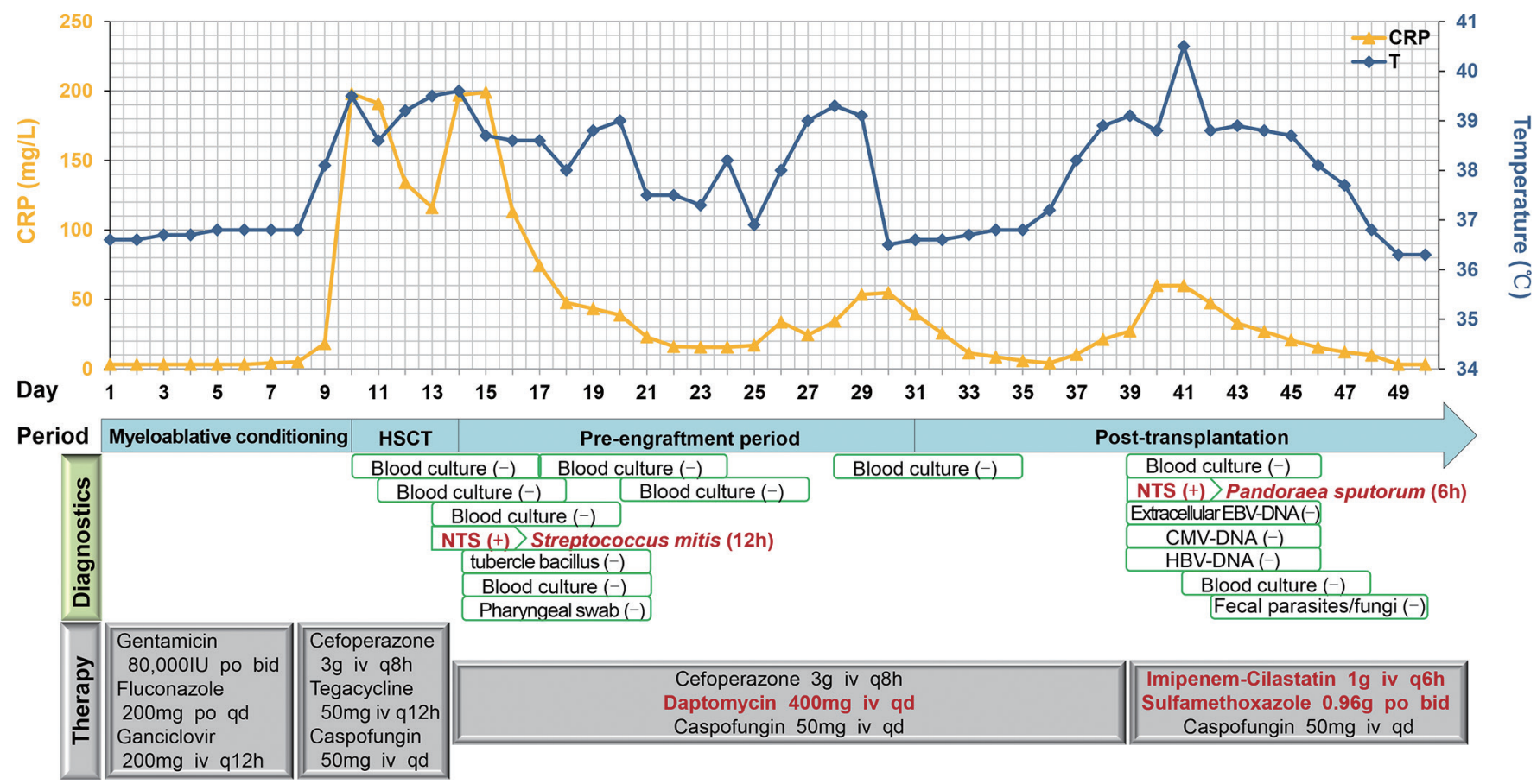

Figure S1 Timeline of clinical management progression of case 1 who underwent allo-HSCT. Timing of laboratory diagnostic tests, including conventional culture and nanopore targeted sequencing, and early antimicrobial therapies are depicted. CRP: C-reactive protein; temperature: top body temperature of the day; myeloablative conditioning regimen: etoposide + busulfan + cyclophosphamide + antithymocyte globulin; allo-HSCT: allogeneic hematopoietic stem cell transplantation; NTS: nanopore targeted sequencing; CMV: cytomegalovirus; EBV: Epstein-Barr virus; HBV: hepatitis B virus. 


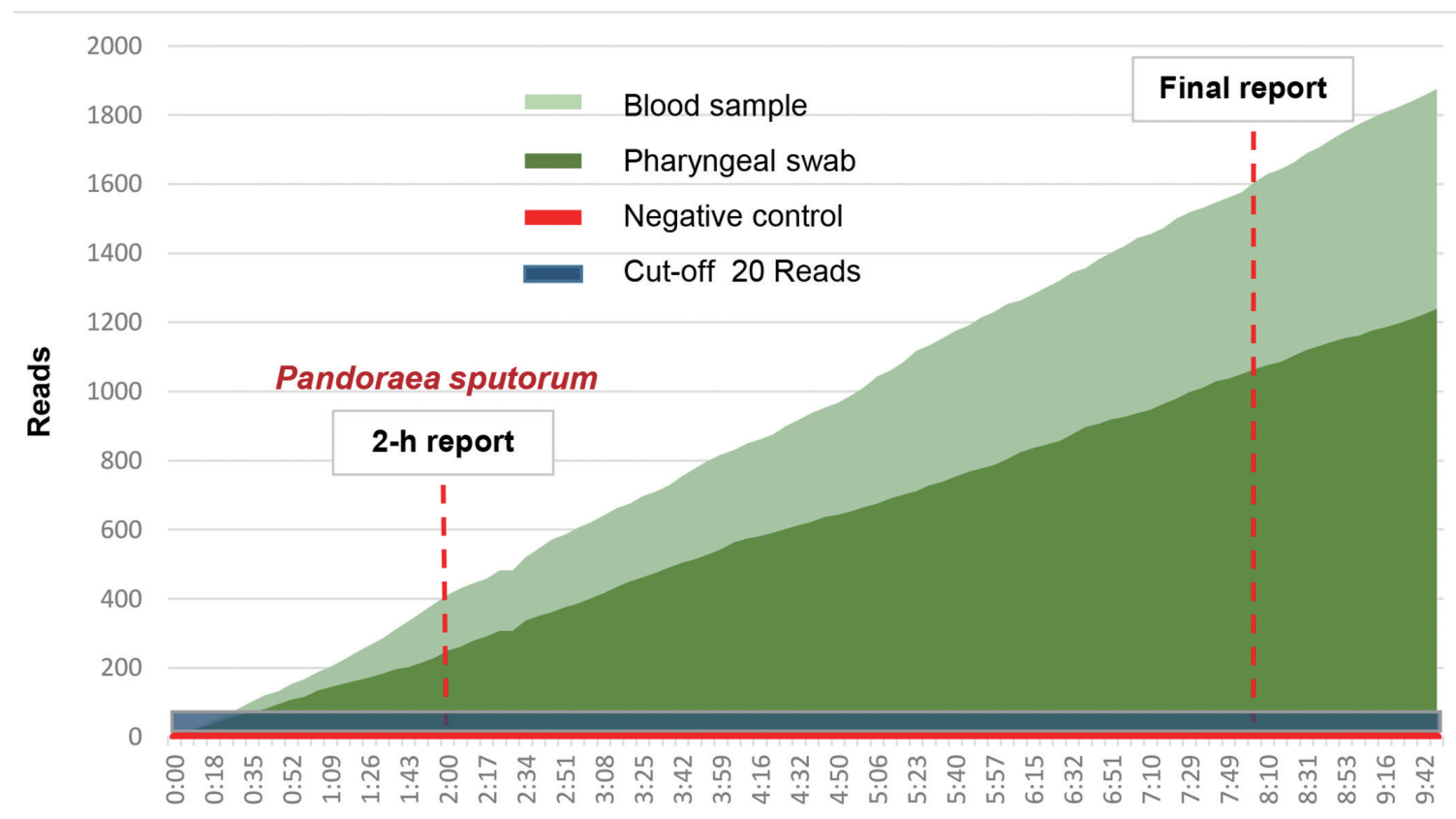

\section{Real-Time Sequencing/Analyzing (hours)}

Figure S2 The second real-time nanopore-targeted sequencing of case 1. Highly abundant, multidrug-resistant Pandoraea sputorum was detected in blood after 25 min of sequencing, as well as in pharyngeal swab after $40 \mathrm{~min}$ of sequencing. The pathogen was report within $2 \mathrm{~h}$ after NTS. The clinical turnaround time was less than 6 hours.

Table S1 Clinical characteristics of the 11 patients with severe infections

\begin{tabular}{|c|c|c|c|c|c|c|c|}
\hline Case & Gender & $\begin{array}{c}\text { Age } \\
\text { (years) }\end{array}$ & $\begin{array}{l}\text { Primary } \\
\text { disease }\end{array}$ & $\begin{array}{l}\text { Treatment for } \\
\text { primary disease }\end{array}$ & Immunosuppressive agents & Symptoms & $\begin{array}{l}\text { WBC }\left(10^{9} / L\right) \\
\text { at infection }\end{array}$ \\
\hline 1 & Female & 15 & ALL & Allo-HSCT & FK506+MTX+MMF+anti-CD25+ATG & $\begin{array}{l}\text { High fever, oral ulcer, } \\
\text { diarrhea }\end{array}$ & 0.02 \\
\hline 2 & Female & 41 & AML & Allo-HSCT & FK506+MTX+MMF+anti-CD25+ATG & High fever & 0.26 \\
\hline 3 & Male & 25 & MAL & Allo-HSCT & FK506+MTX+MMF+anti-CD25+ATG & High fever, oral ulcer & 0.04 \\
\hline 4 & Male & 52 & MDS & Allo-HSCT & FK506+MTX+MMF+anti-CD25+ATG & $\begin{array}{l}\text { High fever, cough, } \\
\text { diarrhea }\end{array}$ & 0.3 \\
\hline 5 & Male & 50 & AML & Allo-HSCT & FK506+MTX+MMF+anti-CD25+ATG & High fever & 0.02 \\
\hline 6 & Male & 17 & ALL & Allo-HSCT & FK506+MTX+MMF+anti-CD25+ATG & High fever & 0.01 \\
\hline 7 & Female & 31 & AML & Allo-HSCT & FK506+MTX+MMF+anti-CD25+ATG & $\begin{array}{l}\text { Low fever, vomiting, } \\
\text { diarrhea }\end{array}$ & 3.77 \\
\hline 8 & Male & 61 & AML & Chemotherapy & None & $\begin{array}{l}\text { High fever, cough, } \\
\text { diarrhea, septic shock }\end{array}$ & 0.92 \\
\hline 9 & Male & 37 & ALL & Allo-HSCT & $\mathrm{CSA}+\mathrm{MTX}+$ anti-CD25 & High fever & 0.01 \\
\hline 10 & Male & 31 & $\begin{array}{l}\text { CML- } \\
\text { AP }\end{array}$ & $\begin{array}{l}\text { Punatinib + } \\
\text { allo-HSCT }\end{array}$ & FK506+MTX+MMF+anti-CD25+ATG & High fever & 0.82 \\
\hline 11 & Male & 49 & ALL & allo-HSCT & FK506+MTX+MMF+anti-CD25+ATG & High fever, oral ulcer & 0.05 \\
\hline
\end{tabular}

AML: acute myeloid leukemia; ALL: acute lymphoblastic leukemia; MAL: mixed-phenotype acute leukemia: MDS: myelodysplastic syndrome; CML-AP: accelerated phase of chronic myeloid leukemia; allo-HSCT: allogeneic hematopoietic stem cell transplantation; WBC: white blood cell; FK506: tacrolimus; MTX: methotrexate; MMF: mycophenolate mofetil; ATG: antithymocyte globulin; CSA: cyclosporine A. 